\title{
Non-syndromic supernumerary premolar tooth buds at a rare site in the mandible
}

\author{
anand marya ${ }^{1}$ and Adith Venugopal ${ }^{2}$ \\ ${ }^{1}$ University of Puthisastra \\ ${ }^{2}$ Saveetha University Saveetha Dental College
}

November 8, 2021

\begin{abstract}
Hyperdontia involving the premolar teeth can be single or multiple or even related to a syndrome. These are frequently encountered in permanent dentition, but their occurrence has ranged between 0.09 to $0.64 \%$ in the general population. These are rarely found between the first and second premolars.
\end{abstract}

\section{Introduction}

Hyperdontia involving the premolar teeth can be single or multiple or even related to a syndrome. Their occurrence has ranged between 0.09 to $0.64 \%$ in the general population ${ }^{1,2}$. A 21-year-old patient with a non-contributory history reported to the clinic with a complaint of spacing in the anterior teeth. On radiographic examination, eumorphic supernumerary premolar crowns were found located between the roots of the mandibular first and second premolars (Figure 1). The patient was completely asymptomatic and had no knowledge regarding the presence of these accessory teeth. A Neumann full-thickness flap was utilized to remove these during the course of orthodontic treatment. A lingual approach is preferred during their removal over the buccal method as it poses less risk of root trauma. The shortcoming of this approach is that although it is better for visualization, it poses a high risk of trauma to the adjacent teeth or nerves. As the premolar was being removed on the right side, there was a fracture of the root apex. On follow-up over the next 1 year, no other complications or secondary tooth germs were observed, and following a period of bone formation, the bone in the affected area normally formed again (Figure 2).

\section{Figure Legends}

Figure 1: Image depicting the supernumerary premolar buds located between the first and second mandibular premolars 


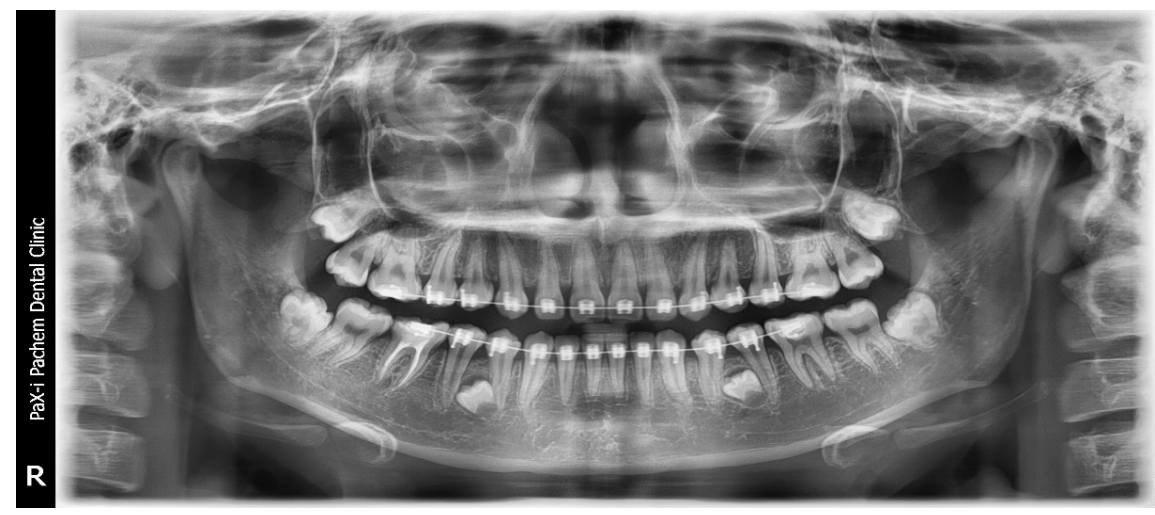

Figure 2: Follow-up panoramic image 1-year after removal of the supernumerary premolars.

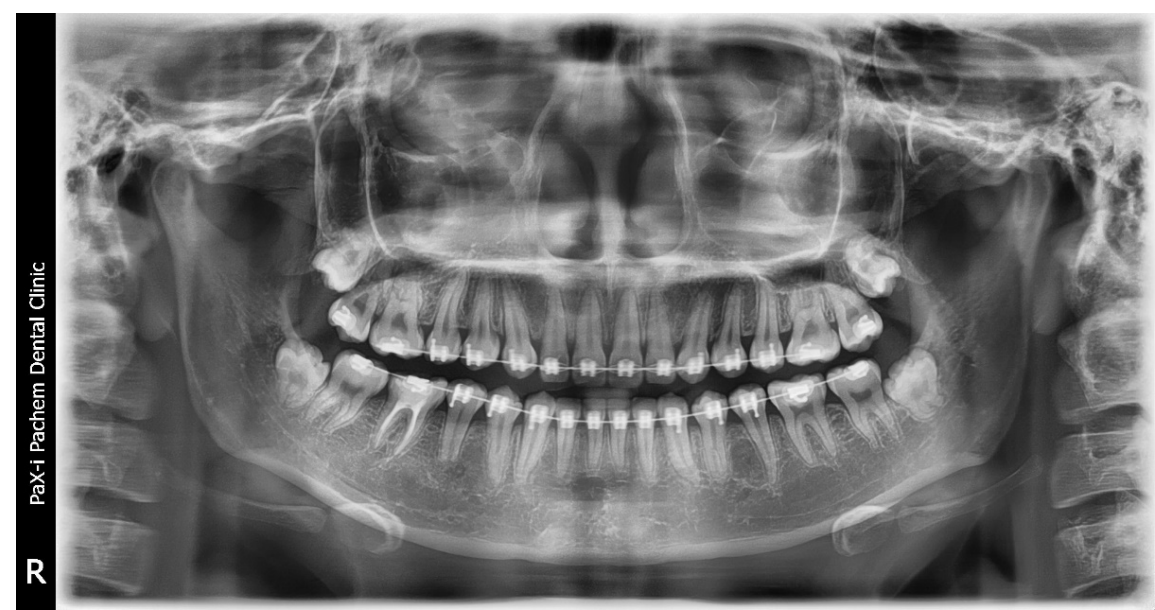

\section{Ethical Statement}

Because this report involves no experiment, ethics approval is waived.

\section{Acknowledgment}

The authors would like to thank the patient for giving consent.

\section{Patient consent}

Written informed consent was obtained from the patient to publish this report in accordance with the journal's patient consent policy

\section{Conflict of Interest}

The authors made no disclosures.

Author contributions: AM: patient treatment AM \& AV: diagnosis and treatment planning AM: manuscript preparation, AM \& AV: review and editing

\section{References}

Stafne E. C. Supernumerary teeth. Dental Cosmos. 1932;74:653-659. 
Rubenstein L. K., Lindauer S. J., Issacson R. J., Germane N. Development of supernumerary premolars in an orthodontic population. Oral Surgery, Oral Medicine, Oral Pathology. 1991;71:392-395. doi: 10.1016/00304220(91)90324-6 